\title{
Tuning Paclitaxel Release Behavior Using Dually- Responsive Nanomicellar Assemblies with Multiple Diselenide/Disulfide Linkages
}

Jingwen Xu ( 4460@sust.edu.cn )

Shaanxi University of Science and Technology Xi\'an Campus: Shaanxi University of Science and Technology https://orcid.org/0000-0002-7055-2826

\section{Xiangji Yan}

Xi'an Jiaotong University

\section{Yue Zhang}

Shaanxi University of Science and Technology Xi\'an Campus: Shaanxi University of Science and

Technology

\section{Kangsheng Tu}

The First Affiliated Hospital of Xi'an Jiaotong University

\section{Wen Shen}

Shaanxi University of Science and Technology Xil'an Campus: Shaanxi University of Science and Technology

\section{Guoliang Li}

Shaanxi University of Science and Technology Xi\'an Campus: Shaanxi University of Science and Technology

\section{Zhaoqing Tian}

Shaanxi University of Science and Technology Xi\'an Campus: Shaanxi University of Science and Technology

\section{Wei Zhao}

the First Affiliated Hospital of Xi'an Jiaotong University

\section{Mingzhen Zhang}

Xi'an Jiaotong University

\section{Research}

Keywords: Diselenide bond, Redox-responsive, Thermal-responsive, Nanomicellar complex, PTX controlled release

Posted Date: October 1st, 2021

DOI: https://doi.org/10.21203/rs.3.rs-936666/v1 
License: (c) (i) This work is licensed under a Creative Commons Attribution 4.0 International License. Read Full License 


\section{Abstract}

Background: There are many reasons that lead to the failure of cancer chemotherapy, such as uncontrolled drug release, low drug utilization, and severe side effects.

Methods: To overcome these obstacles, we designed two kinds of copolymers with thermal and redoxresponsive properties containing multiple diselenide/disulfide bonds polyethylene glycol -altdiselenodipropionate/disulfhydryldipropionate-b-poly(N-isopropylacrylamide) (abbreviated as PEG-altDSeDP-b-PNIPAM and PEG-alt-DSDP-b-PNIPAM) by alternative esterification and following atom transfer radical polymerization. Afterward, these prepared copolymers were mixed with the mass ratio of 8:0, 5:3, 3:5, 0:8 (denoted as S1, S2, S3, and S4, respectively), and self-assembled with paclitaxel (PTX) to obtain PTX-loaded S1, S2, S3, and S4 nanomicellar assemblies, aiming to realize PTX controlled and on-demand release.

Results: The chemical structures of these two copolymers were characterized by gel permeation chromatography (GPC), indicating eight diselenide/disulfide linkages and eight PEG units were contained in these copolymers. Moreover, the thermal-responsive property was detected by UV-vis spectroscopy, meanwhile, the redox-responsive property was observed by TEM in the presence of $10 \mathrm{mM}$ glutathione (GSH). We found that $76.90 \%$ of PTX was released from S1 nanomicelles within $23 \mathrm{~h}$. In contrast, this percentage decreased to $64.53 \%$ for $S 4$ nanomicelles even the incubation time prolonged to $82 \mathrm{~h}$, indicating explosive and slow release behaviors of S1 and S4 nanomicelles, respectively. In addition, gradually decreased fluorescence intensity around the cellular nucleus was occurred from S1 to S4 orderly, which was consistent with cellular uptake and in vivo anti-tumor experiments.

Conclusion:This work not only provides a strategy for the controlled and effective release of PTX, but also improves drug bioavailability in cancer treatment.

\section{Background}

Nowadays, there are many reasons that lead to the failure of cancer chemotherapy, such as uncontrolled drug release, low drug utilization, and severe side effects. Many strategies were developed to overcome these obstacles confronting in cancer chemotherapy. Among them, nanovehicles composed of stimulitriggered block copolymers with biocompatibility and biodegradability offered promising channels for hydrophobic anticancer drugs delivery. [1-3] Although these carriers are constructed based on the abnormal physiological signals at the tumor site such as $\mathrm{pH}$, temperature, enzyme, hypoxia, and redox components, etc., which still produce severe defects including retard, explosive, lagged, and even uncontrolled release. [4-8] Tumors at different stages showed varied demand for drugs, but no markedly discriminate release behavior of existing nanovehicles can result in low drug utilization and severe side effects. [9] In previous studies, single stimuli-responsive drug nanovehicles were designed but possessed unsatisfactory release behavior due to limited response functional groups contained and inaccurate controllable capacity. Further, the nanocarriers with dual and even multiple responsiveness through 
synergistic effects were reported. [10-12] However, the correlation between the two or multiple response mechanisms and drug-controlled behavior remains unclear. Thus, there is of great significance to investigate the relevance and to realize controlled and on-demand release for cancer treatment. In 2017, Siepmann et al. accelerated drug release rate by compression of milled drug-PLGA/ Poloxamer/PEO powder blends, while the contraction of drug-loaded PLGA-Poloxamer/PEO microparticles could retard release. This phenomenon was caused by the welling/disintegration behavior of the implants upon exposure to the release medium. [13] In addition, a multifunctional polymeric vesicular nanovehicle (PVN) composed of stimuli-responsive micelles was constructed by Kim's group for co-delivery of doxorubicin (DOX) and IR780 iodide. Further, the controlled release of DOX/IR780 iodide was illustrated. [14]

Within solid tumors, the concentration of reduced glutathione (GSH) in the cytosol (2-10 mM) is much higher than that in extracellular fluids $(2-20 \mu \mathrm{M}),[15,16]$ which can induce the cleavage of diselenide and disulfide bond, $[17,18]$ leading to disassembly of nanocarriers and drug release subsequently. In addition, reactive oxygen species (ROS) play a pivotal role in controlling drug release and are identified to be related to many diseases, including cancer. As the most stable and ample nonradical ROS in cells, the concentration of $\mathrm{H}_{2} \mathrm{O}_{2}$ in normal tissue is approximate $20 \mathrm{nM}$, while the concentration of $\mathrm{H}_{2} \mathrm{O}_{2}$ can be up to $50-100 \mu \mathrm{M}$ in tumor site. [19] Therefore, the apparent ROS concentration discrepancy, an abnormal physiological condition, showed great potential for cancer treatment by distinguishing cancerous tissue from the normal .

Selenium (Se) is one of the necessary trace elements that can affect physiological activitiesof human body. Both Senanoparticles and nano-Se were reported due to its unique properties including antibacterial, antiinflammation and antitumor, etc. $[20,21]$ In addition, considering the existence of ultrahigh concentration of GSH, diselenide contained polymers have proven to be an excellent candidate as redox-induced nanocarriers, which can be reduced to selenol in reduced ambient and oxidized to selenic acid under oxidative environment. [22, 23] Due to the fewer responsive linkage contained, the release was still sub-par and needed to be improved. As one of the redox-sensitive groups, disulfide bond has higher bonding energy $\left(240 \mathrm{KJ} \mathrm{mol}^{-1}\right)$ than that of diselenide bond $\left(176 \mathrm{KJ} \mathrm{mol}^{-1}\right)$, it can be referred that the disulfide bond containing polymers have higher stability, slower response and release rate, which has been confirmed by our previous work. $[24,25]$ In some instances, slow drug release is what we pursue. [26]

Taken together, such nanovehicles with the following features are expected to design: (1) multiple responsive linkages should be contained for adjustable drug release; (2) good stability for hydrophobic anticancer drug protection; (3) good biocompatibility to circumvent severe side effects. we designed two kinds of copolymers with thermal and redox-responsive properties containing multiple diselenide/disulfide bonds polyethylene glycol -alt- diselenodipropionate/disulfhydryldipropionate-bpoly(N-isopropylacrylamide) (abbreviated as PEG-alt-DSeDPA-b-PNIPAM and PEG-alt-DSDPA-b-PNIPAM) by alternative esterification and following atom transfer radical polymerization. (Scheme 1(A)) Afterward, these prepared copolymers were mixed in line with the mass ratio of 8:0, 5:3, 3:5, 0:8 (denoted as S1, S2, 
S3, and S4, respectively), and self-assembled with paclitaxel (PTX) to obtain PTX-loaded S1, S2, S3, and S4 nanomicellar assemblies, aiming to realize PTX controlled and on-demand release.

The chemical structures of these copolymers were characterized by gel permeation chromatography (GPC), indicating eight diselenide/disulfide linkages and eight PEG units contained in these copolymers. Moreover, the thermal-responsive property was detected by UV-vis spectroscopy, and the redox-responsive property was observed by TEM in the presence of $10 \mathrm{mM}$ glutathione (GSH). We found that $76.90 \%$ of PTX was released from S1 nanomicelles within $23 \mathrm{~h}$. In contrast, this percentage decreased to $64.53 \%$ for S4 nanomicelle even the incubation time prolonged to $82 \mathrm{~h}$, indicating explosive and slow release behaviors of S1 and S4 nanomicelles, respectively. In addition, gradually decreased fluorescence intensity around the cellular nucleus was occurred from S1 to S4 orderly, which was consistent with cellular uptake and in vivo anti-tumor experiments. Taken together, this work not only provides a strategy for the controlled and effective release of PTX, but also improves drug bioavailability in cancer treatment. (Scheme 1(B))

\section{Materials And Methods \\ 2.1 Materials}

Selenium powder (99.99\%, metals basis), 3-chloropropionic acid (CPA, 98\%) and N-isopropyl acrylamide (NIPAM, 98\%), 2-bromoisobutyryl bromide (BiBB, 98\%), Cuprous cholride (CuCl, 99\%), tris((N,Ndimethylamino)ethyl)amine (ME $\mathrm{M}_{6}$ TREN, 99\%), paclitaxel (PTX, 98\%) and 3,3'-dithiodipropionic acid (DTDPA, 99\%) were provided by Macklin biochemical technology co., LTD. (Shanghai, China). All the chemicals were provided by Sinopharm Chemical Reagent Co., Ltd. (Shanghai, China).

\subsection{Cells and animals}

The murine fibroblast cell line L929, human colon cancer cell line Colon-26 and murine breast tumor cell line 4T1 were purchased from Fanchang Biotechnology Co. Ltd. (Shaanxi, China) and incubated in RPMI 1640 supplemented with $10 \%$ hyclone fetal serum at $37^{\circ} \mathrm{C}$ under $5 \%$ of $\mathrm{CO}_{2}$ atmosphere. Cells in exponential growth phase were selected for further studies.

The male Balb/c mice ( $20 \pm 2 \mathrm{~g})$ were offered by Laboratories Animal Center of Xi'an Jiaotong University (Shaanxi, China) and employed as the model bearing 4T1 tumor.

\subsection{Synthetic procedure}

The diselenide/disulfide bond containing dually responsive copolymers PEG-alt-DXDP-b-PNIPAM (X = Se, $S)$ were synthesized via alternative esterification and ATRP polymerization, the specific synthetic procedure was exhibited in Scheme 1(A). 


\subsubsection{Synthesis of polyethylene glycol-alt- diselenodipropionate/disulfhydryldipropionate}

At first, 3,3'-diselenodipropionic acid (DSeDPA) was synthesized according to the method reported previously.[24] Then, polyethylene glycol-alt-diselenodipropionate/disulfhydryldipropionate (denoted as PEG-alt-DXDP, X represent Se, S) was synthesized by alternative esterification. Taking PEG-alt-DSeDP as an example, DSeDPA (0.304 g, $1 \mathrm{mmol})$, PEG $\left(\mathrm{M}_{n}=2000 \mathrm{~g} \mathrm{~mol}^{-1}, 2 \mathrm{~g}, 1 \mathrm{mmol}\right)$ and 4-

dimethylaminopyridine (DMAP, $0.1222 \mathrm{~g}, 1 \mathrm{mmol}$ ) were dissolved into a mixed solvent composed of DCM and DMF $(v / v=1 / 1,10 \mathrm{~mL})$, which was kept in the ice bath for $3 \mathrm{~h}$ and then added dropwise a solution of $\mathrm{N}, \mathrm{N}^{\prime}$-dicyclohexylcarbodiimide (DCC, $0.4123 \mathrm{~g}, 2 \mathrm{mmol}$ ) in above mixed solvent $(4 \mathrm{~mL})$ via constant pressure funnel within 30 min. Afterwards, the reaction was carried out with $\mathrm{N}_{2}$ inletting at $25^{\circ} \mathrm{C}$ for $24 \mathrm{~h}$. Insoluble $\mathrm{N}, \mathrm{N}^{\prime}$-dicyclohexylurea (DCU) was filtrated to collect supernatant, the canary solid was harvested after rotary evaporation, multiple precipitations over the excess ether, and vacuum drying at $40^{\circ} \mathrm{C}$ for 24 h, affording PEG-alt-DSeDP (Yield: 68\%).

\subsubsection{Synthesis of bromine terminated macromolecular initiator}

The bromine terminated macromolecular initiator Br-PEG-alt-DXDP was synthesized through acylbromination. For Br-PEG-alt-DSeDP, a solution of PEG-alt-DSeDP $(1.856 \mathrm{~g}, 0.1 \mathrm{mmol})$ in anhydrous DCM $(20 \mathrm{~mL})$ in a $100 \mathrm{~mL}$ bottle was shifted in the ice bath. At that time, TEA $(20.24 \mathrm{mg}, 0.2 \mathrm{mmol})$ and DMAP $(12.22 \mathrm{mg}, 0.1 \mathrm{mmol})$ were added under nitrogen atmosphere to the bottle, and DCM solution $(5 \mathrm{~mL})$ of BiBB ( $34.5 \mathrm{mg}, 0.15 \mathrm{mmol}$ ) was added dropwise in the above system via constant pressure funnel within $1 \mathrm{~h}$ afterwards. The reaction was left for $24 \mathrm{~h}$ at $25^{\circ} \mathrm{C}$ with stirring. The product was isolated after removing quaternary ammonium salt and solvent by filtration and rotary evaporation, respectively, followed by multiple precipitations in superfluous ice ether, affording Br-PEG-alt-DSeDP (Yield: $50 \%$ ).

\subsubsection{Synthesis of dually responsive copolymer PEG-alt- DXDP-b-PNIPAM}

The dually sensitive copolymer PEG-alt-DXDPA- $b$-PNIPAM was synthesized through ATRP polymerization and in line with the feed ratio of macromolecular initiator/NIPAM/ME ${ }_{6} \mathrm{TREN} / \mathrm{CuCl}=1 / 150 / 1 / 1$. Taking the preparation of PEG-alt-DSeDP- $b$-PNIPAM as an example, macromolecular initiator $(0.187 \mathrm{~g}, 0.01$ $\mathrm{mmol})$ and NIPAM $(0.17 \mathrm{~g}, 1.5 \mathrm{mmol})$ were dissolved in the mixed solvent of deionized water and DMF $(v / v=4 / 6,4 \mathrm{~mL})$, then $\mathrm{ME}_{6} \operatorname{TREN}(6.12 \mathrm{mg}, 0.03 \mathrm{mmol})$ and $\mathrm{CuBr}(2.87 \mathrm{mg}, 0.02 \mathrm{mmol})$ were quickly introduced in the above mixture. "Freeze-pump-thaw" operation was administrated thrice to expel the air and moisture in the system. Hereafter, the polymerization was left at $25^{\circ} \mathrm{C}$ for $24 \mathrm{~h}$. The mixture was passed through a neutral alumina column to remove the copper catalyst. The liquor was collected, concentrated by rotary evaporation, following dialysis against deionized water for $72 \mathrm{~h}$, yielding white solid after freeze-drying. (Yield: $36.7 \%$ ) 
Similarly, PEG-alt-DTDP, macromolecular initiator Br-PEG-alt-DTDP and dually responsive PEG-alt-DTDP- $b$ PNIPAM with the same feed ratio were also prepared using the same procedures as above.

\subsection{Generation of (PTX encapsulated) nanomicellar assembly}

The nanomicellar assembly S1, S2, S3 and S4 were composed of $40 \mathrm{mg}$ of the two dually responsive copolymers PEG-alt-DSeDP- $b$-PNIPAM and PEG-alt-DSDP- $b$-PNIPAM in line with different mass ratios of 8:0, 5:3, 3:5 and 0:8, respectively. The mixed copolymer powder was dissolved in DMF (10 mL) with stirring and then transferred into a dialysis bag (MWCO: $10 \mathrm{kDa}$ ) to dialyze against deionized water for 72 $h$ to remove solvent and induce the generation of micelles. The fresh water was replaced within predetermined time intervals. At the end of dialysis, the dialysate was collected in a $25 \mathrm{~mL}$ volumetric flask for further measurements.

The model anticancer drug PTX was selected for the preparation of drug loaded nanomicellar assembly. In detail, $40 \mathrm{mg}$ of copolymer powder with the same mass ratio as mentioned above and $10 \mathrm{mg}$ of PTX were dissolved in DMF (10 mL) till cloudy, then the mixture was transferred to a dialysis bag (MWCO: 10 $\mathrm{kDa})$ and immersed in deionized water $(2000 \mathrm{~mL})$. The water was updated at predetermined time intervals. The dialysate was gathered and centrifuged at low speed for removal of unpackaged PTX, the supernatant was lyophilized, and the resulting powder was stored at $4{ }^{\circ} \mathrm{C}$. PTX loading content (LC) and encapsulation efficiency (EE) can be calculated according to the following formula:

LC $(\%)=($ mass of PTX in nanomicelles/mass of PTX-encapsulated nanomicelles $) \times 100 \%(1)$

EE $(\%)=($ mass of PTX in nanomicelles/mass of PTX mixed initially $) \times 100 \%(2)$

Here, mass of PTX in micelles was detected from UV-vis absorbance of the DMF solution of prepared nanomicelles in line with the PTX calibration formula:

$C\left(\mathrm{mg} \mathrm{L}^{-1}\right)=(\mathrm{A}-0.0128) / 0.39082\left(\mathrm{R}^{2}=0.9982\right)(3)$

Where $\mathrm{C}$ denote the concentration of PTX in standard solution, and A represent the absorbance at 210 $\mathrm{nm}$.

\subsection{Characterizations}

${ }^{1} \mathrm{H}$ NMR spectra were recorded on a $400 \mathrm{MHz}$ Bruker Avance Spectrometer (Bruker, Germany) at $25^{\circ} \mathrm{C}$ using DMSO-d6, $\mathrm{CDCl}_{3}$ as solvent, respectively, tetramethylsilane (TMS) was selected as internal reference. Fourier transform infrared spectra (FTIR) were recorded on an EQUINX55 Fourier transformation infrared spectrophotometer (Bruker Corp., Germany) using potassium bromide pellets. The experimental molecular weight of the copolymers was calculated from ${ }^{1} \mathrm{H}$ NMR spectra, moreover, the molecular weight $\left(\mathrm{M}_{w}, \mathrm{M}_{n}\right)$ and polydispersity index (PDI, $\left.\mathrm{M}_{w} / \mathrm{M}_{n}\right)$ of copolymers was detected by gel permeation chromatography (Waters Corp., Milford, USA) system equipped with a Waters 515 pump and 
a Waters 2410 refractive index detector. The copolymers $(4 \mathrm{mg})$ were dissolved THF $(2 \mathrm{~mL}$, chromatographically pure) completely under stirring and filtered through Teflon filter $(0.22 \mu \mathrm{m}$, organic

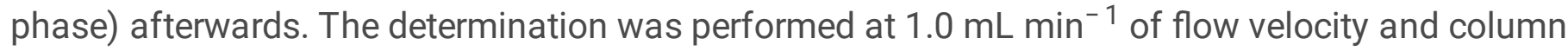
temperature was $35^{\circ} \mathrm{C}$.

To assess critical micelles concentration (CMC) of prepared nanomicelles, the measurements were administrated on a FluoroMax-4 spectrofluorophotometer (HORIBA Scientific, USA) and using pyrene as a fluorescence probe. Specifically, the acetone solution $(4.8 \mu \mathrm{L})$ of pyrene $\left(5 \times 10^{-4} \mathrm{M}\right)$ was added in sample tube and volatilized for $2 \mathrm{~h}$ in dark, following the addition of each of $4 \mathrm{~mL}$ nanomicellar solution with different concentration gradient $\left(5 \times 10^{-5}\right.$ to $\left.5 \times 10^{-1} \mathrm{mg} \mathrm{mL}^{-1}\right)$ with stirring overnight. The excitation wavelength was $331 \mathrm{~nm}$ and fluorescence intensity was collected at $350 \sim 550 \mathrm{~nm}$. All the slit widths were $2 \mathrm{~nm}$. CMC was calculated according to the onset of the decrease in the curves plotted from fluorescence intensity ratio at 383 and $372 \mathrm{~nm}\left(\mathrm{I}_{383} / \mathrm{I}_{372}\right)$ versus the logarithm of nanomicellar concentrations.

The hydrodynamic diameter $\left(\mathrm{D}_{h}\right)$, size distribution (polydispersity index, PDI), and Zeta potentials $(\xi)$ of nanomicelles were measured by a dynamic light scattering (DLS, BI-90Plus, America) instrument. Each measurement was performed thrice at $25^{\circ} \mathrm{C}$, and an average value was reported.

The photo of nanomicelles was captured to observe the morphologies and size dispersion in a transmission electron microscopy (TEM, JEM-2100, Electron Crop., Japan) at an accelerating voltage of $200 \mathrm{keV}$. The sample was prepared by transferring a drop of nanomicellar solution $\left(10 \mu \mathrm{L}, 100 \mathrm{mg} \mathrm{L}^{-1}\right)$ on a carbon film with air-drying before observation.

The UV-vis absorbance and transmittance of nanomicelles and PTX were recorded on an UV-vis spectrophotometer (UV-6100S, Mapada, Shanghai).

The celluar absorbance at $570 \mathrm{~nm}$ was recorded using a microplate reader (Thermo Fisher Scientific, USA).

\subsection{Thermal, redox-responsive behaviors of nanomicellar assembly}

The thermal-responsive of nanomicellar assembly $\left(100 \mathrm{mg} \mathrm{L}^{-1}\right)$ was reflected by the UV-vis transmittance change in the temperature ranged from 20 to $60^{\circ} \mathrm{C}$. The lower critical solution temperature (LCST) of nanomicellar solution was calculated from the half of transmittance decrease versus the corresponding temperature.

To investigate the redox-responsive, the size changes of nanomicellar solution $\left(4 \mathrm{~mL}, 100 \mathrm{mg} \mathrm{L}^{-1}\right)$ treated with $10 \mathrm{mM} \mathrm{GSH}$ or DTT within $0.5,4$ or $24 \mathrm{~h}$ under stirring at $37^{\circ} \mathrm{C}$ were measured by DLS. After $24 \mathrm{~h}$, the morphology of the above nanomicelles was observed by TEM. Similarly, $100 \mu \mathrm{M}$ of $\mathrm{H}_{2} \mathrm{O}_{2}$ was 
employed for oxidizing nanomicelles under the same conditions, and the size change and morphology were also investigated.

\subsection{Thermo and redox induced PTX release in vitro}

Freeze-dried PTX-encapsulated nanomicellar powder $(4 \mathrm{mg})$ was suspended in PBS solution $(4 \mathrm{~mL}, \mathrm{pH}$ $5.6,7.4$ ) entirely and then shifted into a dialysis tube (MWCO: $2 \mathrm{kDa}$ ) into a beaker containing $200 \mathrm{~mL}$ of the corresponding pH value PBS with $10 \mathrm{mM} \mathrm{GSH}$, DTT or $100 \mu \mathrm{M} \mathrm{H}_{2} \mathrm{O}_{2}$ at 37 or $42^{\circ} \mathrm{C}$ for $82 \mathrm{~h}$. The beaker was transferred to a shaking bed at $100 \mathrm{rpm}$. At predetermined time intervals, $4 \mathrm{~mL}$ of dissolution medium were withdrawn from beaker and supplemented with isometric fresh medium. The accumulated drug release amount was assessed from the absorbance of dialysate extracted by a UV-vis spectrophotometer at $210 \mathrm{~nm}$. The amount of released PTX was calculated using the following formula:

Cumulative PTX release $(\%)=M_{t} / M_{o} \times 100 \%$ (4)

Where $\mathrm{M}_{t}$ and $\mathrm{M}_{o}$ denote the mass of PTX at time $t$ and initial moment in nanomicelles, respectively.

\subsection{In vitro cytotoxicity assay}

The cytotoxicity of the prepared blank and PTX-entrapped nanomicelles against L929 cells was evaluated via MTT assay. L929 cells were seeded at a density of $5 \times 10^{4}$ cells per well on a 96-well plate with DMEM $(180 \mu \mathrm{L})$ containing $10 \%$ hyclone fetal serum, then the cells were cultured for $24 \mathrm{~h}$ at $37^{\circ} \mathrm{C}$ under the atmosphere of $95 \%$ air- $5 \% \mathrm{CO}_{2}$. Afterward, the medium was replaced by fresh medium, the blank, PTX-entrapped micelles with different concentrations from 1 to $500 \mu \mathrm{g} \mathrm{mL}^{-1}$, and free PTX with the concentration of $0.1 \sim 20 \mu \mathrm{g} \mathrm{mL}^{-1}$, respectively. The cells were suspended to incubate for $24 \mathrm{~h}$. At that point, L929 cells were rinsed by pH 7.4 PBS thrice and replenished with fresh DMEM and MTT stock solution $\left(20 \mu \mathrm{L}, 5 \mathrm{mg} \mathrm{mL}^{-1}\right)$. After $4 \mathrm{~h}$ incubation, DMSO $(120 \mu \mathrm{L})$ was pipetted into deposits with shaking at $25^{\circ} \mathrm{C}$ for $10 \mathrm{~min}$. Meanwhile, L929 cells were cultured in a fresh medium under the same conditions as the negative control. Finally, the absorbance at $570 \mathrm{~nm}$ was recorded on a microplate reader (Thermo Fisher Scientific, USA) to viability according to the following equation:

Cell viability $(\%)=O D_{\text {Sample }} / O D_{\text {Control }} \times 100 \%$ (5)

where the $\mathrm{OD}_{\text {Sample }}$ and $\mathrm{OD}_{\text {Control }}$ were the optical density (OD) of samples and negative controls, respectively. The Student's t-test proceeded to verify the significance of any statistical differences, and all quantitative data were shown as the form of mean \pm SD value.

\subsection{Celluar uptake}

Free PTX and PTX-encapsulated S1 S4 nanomicelles were seeded in a 6-well plate containing $1 \times 10^{5}$ Colon- 26 cells per well and cultured. After $24 \mathrm{~h}$, the initial medium was removed, following the supplemention of new medium with $30 \mu \mathrm{g} \mathrm{ml}^{-1}$ of PTX and incubation for $6 \mathrm{~h}$. The cells were fixed by $4 \%$ paraformaldehyde and rinsed using PBS thrice. The cytoskeleton, nucleus and nanomicelles were labeled 
with phalloidin-FITC, DAPI and Dil, respectively. The qualitative analysis of celluar uptake was performed using a confocal laser scanning microscope (CLSM, Nikon A1, Japan).

Colon 26 cells were treated with free PTX and PTX-loaded S1 S4 nanomicelles and cultured for $6 \mathrm{~h}$, followed by washing with PBS, digestion with trypsin, centrifugation and celluar collection. The red fluorescence intensity of nanomicelles was detected to quantitatively evaluate intracellar PTX content through flow cytometer.

\subsection{Cell apoptosis}

Colon 26 cells were seeded in 6-well plates with cell slides $\left(1 \times 10^{5}\right.$ cells per well) and cultured for $24 \mathrm{~h}$, then the cells were rinsed with PBS and cultured with free PTX, PTX-loaded S1 S4 nanomicelles at 30 $\mu \mathrm{g} \mathrm{ml}^{-1}$ of PTX for $24 \mathrm{~h}$. Then the cells were washed with PBS. As a comparison, the cells were cultured in RPMI 1640 medium as control. Afterwards, the cells were washed with PBS, digested with trypsin, resuspended in PBS and stained using Annexin V-FITC and propidium iodide (PI). The cells apoptosis was monitored through flow cytometry.

\subsection{In vivo anticancer efficacy}

Male Balb/c mice were intravenously injected with $100 \mu \mathrm{L}$ of $4 \mathrm{~T} 1$ cell suspension containing $1 \times 10^{6}$ cells per mouse. when the tumor sizes reached about $100 \mathrm{~mm}^{3}$, mice were randomly divided into 5 groups $(\mathrm{n}=$ 3) and i.v. injected with free PTX, PTX-encapsulated S1 S4 nanomicelles at a PTX dose of $10 \mathrm{mg} \mathrm{kg}^{-1}$ on days $0,3,6,9,12,15$. Mice were treated with $0.9 \%$ normal saline and PBS as control groups. The change of mice body weight and tumor volume was monitored every three days. The tumor size was estimated using the following formula: $V=a b^{2} / 2$. Where $a, b$ represent the longest and the shortest dimension of tumor, respectively.

\subsection{Histological analysis}

On day 15 , all treated mice were sacrificed and the major organs (heart, liver, spleen, lung, kidney) were collected and immersed in 10\% PBS-formaldehyde solution, and embedded in paraffin afterwards. The obtained major organs were sectioned into slices with $5 \mu \mathrm{m}$ of thickness after deparaffinization, followed by staining with hematoxylin and eosin (H\&E) for subsequent histological study through a Zeiss microscope (PA, USA).

\subsection{Statistical analysis}

One-way and two-way analyses of variance (ANOVA) and t-tests were used to determine statistical significance $\left({ }^{\star} p<0.05,{ }^{\star \star} p<0.01,{ }^{* \star} p<0.001\right)$, $p$-value less than 0.05 was considered statistically significant. 


\section{Results And Discussion}

\subsection{Synthesis and characterization of PEG-alt-DXDP- $b$ - PNIPAM}

The dually responsive copolymers PEG-alt-DXDP- $b$-PNIPAM $(X=S e, S)$ with diselenide/disulfide linkages were synthesized via alternative esterification and ATRP polymerization.

The chemical structures of copolymers and their intermediates were confirmed by ${ }^{1} \mathrm{H}$ NMR and FTIR spectra. The results were shown in Fig. 1(a-c) and Figure S1-S3. ${ }^{1} \mathrm{H}$ NMR and FTIR spectra of DSeDPA as shown in Figure $\mathrm{S} 1$ indicated that the chemical shifts of $-\mathrm{SeCH}_{2} \mathrm{CH}_{2},-\mathrm{SeCH}_{2} \mathrm{CH}_{2}$ - and $-\mathrm{CH}_{2} \mathrm{CH}_{2} \mathrm{COOH}$ emerge at 2.85, 3.10, and $12.37 \mathrm{ppm}$, respectively. In addition, the characteristic peaks at 527, [25] 797, $1700,2894-2944$ and $3330-3630 \mathrm{~cm}^{-1}$ were successively attributed to $-\mathrm{C}-\mathrm{Se},-\mathrm{Se}-\mathrm{Se},-\mathrm{C}=\mathrm{O},-\mathrm{C}-\mathrm{H}$ vibration band and $-\mathrm{OH}$ in $-\mathrm{COOH}$. After PEG introduction, the peaks at 2.85, 3.10 ppm still existed. By contrast, almost no difference could be observed except for the chemical shifts of at 2.90, $2.75 \mathrm{ppm}$ in Figure $\mathrm{S} 3(\mathrm{a})$ ascribed to $-\mathrm{SCH}_{2} \mathrm{CH}_{2},-\mathrm{SCH}_{2} \mathrm{CH}_{2}$, respectively, chemical shifts of methane protons in PEG appear at 3.65, $4.25 \mathrm{ppm}$, suggesting the formation of PEG-alt-DXDP. Further, the preparation of PEG-altDSeDP was also proved by the characteristic peaks including $527 \mathrm{~cm}^{-1}$ for $-\mathrm{C}-\mathrm{Se}, 797 \mathrm{~cm}^{-1}$ for -Se-Se-, $1112 \mathrm{~cm}^{-1}$ for $-\mathrm{C}-\mathrm{O}-\mathrm{C}-, 1738 \mathrm{~cm}^{-1}$ for ester $-\mathrm{C}=0,2892 \mathrm{~cm}^{-1}$ for $-\mathrm{C}-\mathrm{H}$ vibration and $3293-3666 \mathrm{~cm}^{-1}$ for $-\mathrm{OH}$ belonged to free terminated $-\mathrm{COOH}$ in Figure $\mathrm{S} 2(\mathrm{~b})$. The position of characteristic peaks of PEG-altDSDP was similar to PEG-alt-DSeDP, as shown in Figure S3(b).

Some chemical shifts including $-\mathrm{CH}_{2}$ - repeated units at $3.65 \mathrm{ppm}$ in $\mathrm{PEG},-\mathrm{CH}_{2}-$ near to diselenium bond at 2.85 and $3.10 \mathrm{ppm},-\mathrm{CH}_{2}-$ connected with PEG at $4.25 \mathrm{ppm}$, methyl proton in $-\mathrm{CH}\left(\mathrm{CH}_{3}\right)_{2}$ belonged to NIPAM at $1.17 \mathrm{ppm},-\mathrm{CH}_{2}-$ in main chains at $1.36-1.74 \mathrm{ppm}$, methyne proton in $-\mathrm{CH}\left(\mathrm{CH}_{3}\right)_{2}$ at $4.03 \mathrm{ppm}$ and amide proton at $6.48 \mathrm{ppm}$ are clearly visible in Fig. 1(a), indicating the formation of PEG$a l t$-DSeDP-b-PNIPAM. Similarly, almost no significant difference was observed between Fig. 1(a) and 1(b), except the chemical shifts that emerged at 2.90 and $2.75 \mathrm{ppm}$ in Fig. 1(b) that associated with $-\mathrm{CH}_{2}-$ in DSDPA, suggesting the successful synthesis of PEG-alt-DSDP-b-PNIPAM. Afterward, in Fig. 1(c), the characteristic peak of -C-Se, -Se-Se-, -C-O-C- bond in PEG, $-\mathrm{C}=\mathrm{O},-\mathrm{C}-\mathrm{H}$ vibration band appear at 527, $797,1110,1730$ and $2875-2925 \mathrm{~cm}^{-1}$, respectively, in addition, the features peaks at 1550, 1652 and $3072 \mathrm{~cm}^{-1}$ were allocated to the amide II, I and III band, respectively, ester $-\mathrm{C}=0$ appear at $1735 \mathrm{~cm}^{-1}$. Due to the hydrogen bonding association, the peak at $3450 \mathrm{~cm}^{-1}$ was caused by the stretching vibration of - NH- in PNIPAM blocks. The tagged peaks of PEG-alt-DSDP-b-PNIPAM were basically consistent with PEG-alt-DSeDP-b-PNIPAM, further signifying that the formation of the two copolymers.

As an important parameter, molecular weight is of great importance to the properties and applications of copolymers. It was observed that the monomodal and quasi-symmetrical elution curves in Fig. 1(d) after PEG and NIPAM introduction, indicating good control during all synthesis procedures. The products were with high purity for PDI was lower than 1.50. Approximately 8 DSeDPA and 8 DSDPA were esterfied with 8 
PEG, respectively, yielding (PEG-alt-DSeDP) $)_{8}$ and (PEG-alt-DSDP $)_{8}$. It could be calculated that 78 and 91 NIPAM units were copolymerized in (PEG-alt-DSeDP) $)_{8}-b$-PNIPAM ${ }_{78}$ and (PEG-alt-DSDP) $)_{8}-b-$ PNIPAM $_{91}$, respectively. The above results are listed in Table 1.

Table 1

Molecular weight of polymers

\begin{tabular}{|lllll|}
\hline Polymers & \multicolumn{4}{l}{ Molercular weight from GPC } \\
\cline { 2 - 5 } & $\mathbf{M}_{\mathbf{n}}$ & $\mathbf{M}_{\mathbf{w}}$ & PDI & Monomer conversion ratio (\%) \\
\hline PEG-alt-DSeDP & 18,566 & 26,549 & 1.48 & \\
\hline PEG-alt-DSDP & 18,000 & 21,420 & 1.19 & \\
\hline PEG-alt-DSeDP-b-PNIPAM & 27,380 & 35,046 & 1.28 & 52.00 \\
\hline PEG-alt-DSDP-b-PNIPAM & 28,283 & 35,303 & 1.39 & 60.67 \\
\hline
\end{tabular}

\subsection{Self-assemble behavior of copolymers}

In view of hydropathy differences between PEG-alt-DXDP segment and PNIPAM blocks, the nanomicelles with PNIPAM core and PEG-alt-DXDP shell were formed, in which diselenide and disulfide bonds served as linkages on the hydrophobic and hydrophilic interfaces. To identify the generation of nanomicelle, pyrene probe was introduced to assemble with prepared nanomicellar solution with different concentrations $\left(5 \times 10^{-5} \sim 5 \times 10^{-1} \mathrm{mg} \mathrm{L}^{-1}\right)$ for $\mathrm{CMC}$ measurement. The curves of pyrene fluorescence intensity ratio $\mathrm{I}_{383} / \mathrm{I}_{372}$ versus logarithm of nanomicellar concentration were presented in Fig. 2(A). S4 has a lower $\mathrm{CMC}$ value of $37.88 \mathrm{mg} \mathrm{L}^{-1}$ than other nanomicelles, which were ascribed to more hydrophobic NIPAM units contained than that in other copolymers. Thus, S3 and S4 nanomicelles are liable to form at relatively low concentrations and maintain micellar structure during blood circulation, implying the potential as a carrier for drug sustained release. By contrast, S1 and S2 have fewer NIPAM units, leading to higher $\mathrm{CMC}$ value (97.60 and $58.89 \mathrm{mg} \mathrm{L}^{-1}$ ) and weaker protection for PTX. Even so, the rapid response of diselenide bond makes $\mathrm{S} 1$ and $\mathrm{S} 2$ good carrier candidates for explosive drug release.

The enhanced hydrophobicity of cores after PTX encapsulation results in the decreased CMC compared with blank nanomicelles (Table 2 and Figure S4), including 57.91, 44.49, 25.30, and $9.78 \mathrm{mg} \mathrm{L}^{-1}$, corresponding to PTX-loaded S1, S2, S3 and S4, respectively. It was suggested that PTX could be sheltered by PEG-alt-DXDP shell.

Zeta potentials indicated that all nanomicelles were positively charged. High absolute zeta potential values indicated the system stability by the exclusion and dispersion degree of adjacent particles. The higher potentials caused by a fine balance of attraction and repulsion can result in a jarless dispersed system, while the lower potentials indicated destroy and flocculation deposition of the system. [27] It was reported that positively nanomicelles were more likely to be absorbed into the negatively charged cell 
membrane. In this work, zeta potential of nanomicelles was in the range of $24.93 \sim 29.29 \mathrm{mV}$, indicating good stability and the possibility of uptake and internalization into cells.

\subsection{Thermo, redox responsive behaviors of nanomicelles assembly}

As the incorporation of PNIPAM, the LCST of polymers was determined by UV-vis spectrums and shown in Fig. 2(B). The LCST obviously decreased with the increase of NIPAM number and reached 40.83, 38.75, 38.47, and $37.56^{\circ} \mathrm{C}$ corresponding to S4, S3, S2, and S1, respectively. The similar LCST of S3 and S4 nanomicelles was ascribed to the equivalent NIPAM content. When the temperature was above LCST, PNIPAM blocked undergo hydrophilic-hydrophobic conversion and become nanomicellar core. It was noteworthy that all LCSTs were higher than $37^{\circ} \mathrm{C}$, and the prepared nanomicelles were expected to realize temperature controlled release.

To investigate whether the diselenide and disulfide linkages could be cracked under reduced or oxidized physiological microenvironment, the as-prepared S1 and S4 nanomicellar solution was incubated with 10 mM GSH within 0.5, 4, 24 h before DLS measurement, while the nanomicelles were treated without GSH as control. After the reduction of diselenide bond into selenol by GSH, the enhanced hydrophilicity of selenol strengthens the swelling effect of the nanomicellar shell, [28] yielding gradually larger nanomicelles size from 127.1, 143.8 to $254.3 \mathrm{~nm}$ (Fig. 3A(b-d)) along with GSH incubate time, which far exceed $106.2 \mathrm{~nm}$ of nanomicelles without reduction (Fig. 3A(a)). Afterward, diselenide bonds were oxidized to seleninic acid by $100 \mathrm{MM} \mathrm{H}_{2} \mathrm{O}_{2}$, obtaining nanomicelles with $86.9 \mathrm{~nm}$ of average $\mathrm{D}_{h}$ (Fig. 3A(e)), thereby the redox-responsive property was confirmed. Further, the morphologies and size distribution for the above nanomicelles were also observed by TEM and illustrated in Fig. 3(B-E). As exhibited in Fig. 3(B), the anticipated core-shell structure with $60 \mathrm{~nm}$ of average diameter was formed preliminarily. After GSH reduction for $24 \mathrm{~h}$, a large number of alveolate holes were visible in nanomicelles and their size presented a sharp increase to $232 \mathrm{~nm}$ from Fig. 3(C), consistent with the DLS results in Fig. 3(A). However, under the oxidization of $100 \mu \mathrm{M} \mathrm{H}_{2} \mathrm{O}_{2}$, the damaged nanomicelles with blurred boundaries appear at first, then converted into irregular aggregation and even disassemble into tiny debris with the size of about $44 \mathrm{~nm}$ in Fig. 3(D), signifying that the oxidation trigger had indeed lead the diselenide bond cleaved into seleninic acid. It was thereby concluded that $\mathrm{S} 1$ nanomicelles possess the redox-responsive property under $\mathrm{GSH}, \mathrm{H}_{2} \mathrm{O}_{2}$ triggers. The smaller nanomicellar size monitored by TEM than DLS was caused by PEG dehydration during the sample preparation. [29] The core-shell appearance was still maintained, and the selenium granules were squeezed to the margin of nanomicelles. With the introduction of PTX into the nanomicellar core, the size of PTX-loaded S1 nanomicelles increased to 112 $\mathrm{nm}$ in Fig. 3(E) compared with $60 \mathrm{~nm}$ of blank S1 nanomicelles. Moreover, PTX was released under the condition of pH 5.6 PBS containing $10 \mathrm{mM} \mathrm{GSH}$ at $40^{\circ} \mathrm{C}$ (above LCST) for $82 \mathrm{~h}$ as exhibited in Fig. $4 \mathrm{~A}(\mathrm{~b})$. The morphology of PTX-loadedloaded S1 nanomicelles released in pH 5.6 PBS containing $10 \mathrm{mM}$ GSH at $40^{\circ} \mathrm{C}$ (above LCST) for $82 \mathrm{~h}$ was shown in Fig. 3(F), the sharply increased size of the new-formed alveolate core-shell nanomicelles is clearly visible, which might be caused by the continuous reduction 
from GSH and the leftover PTX. Moreover, the jaggy reduction degree yielded the uneven nanomicellar size. Likewise, the changes of $\mathrm{D}_{h}$ and morphology of $\mathrm{S} 4$ nanomicelles in the presence of $\mathrm{GSH}$ and $\mathrm{H}_{2} \mathrm{O}_{2}$ were in agreement with S1 nanomicelles, see in Figure S5.

\subsection{Thermal and redox induced PTX release}

As one of the widely used anticancer drugs in clinic applications, PTX was selected for drug encapsulation and release investigation through dialysis method. The multifactor including hydrogen bonding, electrostatic and hydrophobic interactions between PTX and PNIPAM blocks yield a $13.67 \%$, $11.11 \%, 10.58 \%$ and $12.11 \%$ of LC, $54.63 \%, 40.00 \%, 42.32 \%$ and $48.14 \%$ of EE value for PTX-

loadedloaded S1-S4 micelles, respectively. In fact, there were no obvious differences between both LC and EE for PTX-loaded S1-S4 nanomicelles, on account of the synergistic effects including the same feed ratio of DXDP, PEG and NIPAM during copolymer preparation, as well as the mass ratio between copolymer and PTX. Taking CMC, zeta potentials and other factors into consideration, lyophilized PTX loaded S1 and S4 nanomicelles were selected for further drug release.

Then, the thermo and redox triggered drug release behavior of PTX-loaded S1 and S4 nanomicelles were separately assessed under the condition of (a) pH 7.4 PBS at $37^{\circ} \mathrm{C}$, (b) pH 5.6 PBS containing $10 \mathrm{mM}$ GSH at $40^{\circ} \mathrm{C}$, (c) pH 5.6 PBS containing $100 \mu \mathrm{M} \mathrm{H}_{2} \mathrm{O}_{2}$ at $40^{\circ} \mathrm{C}$ and (d) $\mathrm{pH} 5.6 \mathrm{PBS}$ with $100 \mu \mathrm{M} \mathrm{H}_{2} \mathrm{O}_{2}$ at $40{ }^{\circ} \mathrm{C}$ for $82 \mathrm{~h}$, the results were shown in Fig. 4. Only $32.91 \%$ of PTX was released from $\mathrm{S} 1$ nanomicelles in normal physiological signals from Fig. 4A(a), while $17.17 \%$ of PTX was release from S4 nanomicelles at pH 7.4 (Fig. 4B(a)). Approximately $76.90 \%$ of PTX were released within $23 \mathrm{~h}$ from $\mathrm{S} 1$ nanomicelles after the reduction of $\mathrm{GSH}$ at $42{ }^{\circ} \mathrm{C}$ in Fig. $4 \mathrm{~A}(\mathrm{~b})$, respectively, which was 2.33 times than that in $\mathrm{pH} 7.4$ at $37^{\circ} \mathrm{C}$ and ascribed to the fast demicellization and rearrangement of PTX loaded nanomicelle, together with phase transformation of PNIPAM stimulated by temperature. However, not until $82 \mathrm{~h}$ was PTX released from S4 and reached the peak of $64.53 \%$, suggesting that PTX sustained release has realized in Fig. $4 \mathrm{~B}(\mathrm{~b})$. Meanwhile, the cumulative PTX release was $59.18 \%$ under synergistic trigger of $100 \mu \mathrm{M} \mathrm{H}_{2} \mathrm{O}_{2}$ and temperature, see in Fig. 4A(d). However, the fast and even burst release rate of PTX from S1 nanomicelles in Fig. 4A can be ascribed to only diselenide bond possessed in nanoformulations and its higher bonding energy and fast response mechanism. As expected, the more desirable PTX release performance of the well-prepared S2 and S3 nanomicelles composed of S1 and S4 copolymers than that of pure $\mathrm{S} 1$ and $\mathrm{S} 4$ nanomicelles is illustrated.

\subsection{In vitro cytotoxicity of nanomicelles}

MTT assay was performed for evaluation of the cytotoxicity of free PTX, blank and PTX loaded S1-S4 nanomicelles against L929 and HCT116 cells. The cell viability of the cells cultured with free PTX at a different concentration of $0.01 \sim 20 \mu \mathrm{gL}^{-1}$ and blank, PTXloaded loaded nanomicelles at varied concentrations of $1 \sim 500 \mu \mathrm{g} \mathrm{mL}-1$ for $24 \mathrm{~h}$. IC50 of PTX against L929 cells is $1.5 \mu \mathrm{g} \mathrm{mL}^{-1}$ in Figure S6, and the cell viability of L929 cells treated with blank nanomicelles are over $84 \%$, and some even surpass $100 \%$, which may be ascribed to selenium microelement contained in nanomicelles, thereby having a 
great potential in tissue damages repairing caused by PTX and cell growth to some extent. Moreover, the death of a small number of L929 cells in Fig. 5(B) predicted the negligible cytotoxicity of PTX loaded S1S4 nanomicelles, indicating the biocompatibility of nanoformulations. Due to the presence of diselenide and disulfide bond in the scaffold of copolymers, the combinations of S1 and S4 nanomicelles have a great potential in the recognition of normal and cancerous cells.

\subsection{Cellular uptake and apoptosis}

A confocal laser scanning microscope (CLSM) was selected to qualitatively investigate celluar uptake behavior of the PTX loaded S1-S4 nanomicelles and free PTX. The cell nucleus, scaffold and nanomicelles were separately stained by DAPI, FITC and Dil. Figure 6 exhibits the fluorescence photos of Colon26 cells cultured with free PTX and PTX loaded nanomicelles for $6 \mathrm{~h}$. Since PTX itself is not fluorescent, there was no fluorescence observed in free PTX group. In contrast, Dil-stained nanomicelles with varied red fluorescence could be seen in all groups and mainly accumulated around the nucleus, which is due to the similar size of nanomicelles. It is noteworthy that the fluorescence intensity decreases gradually in the order of S1, S2, S3 and S4, which is caused by the different content of diselenide and disulfide linkages contained in nanomicelles, and resulting in varied amount of PTX released from nanocarriers. loadedWith the regulation of the diselenide linkages, PTX loadedloaded S1-S3 nanomicelles released more PTX, while the S4 nanomicelles containing only disulfide linkages showed a slow release rate.

In addition, the celluar uptake was also confirmed quantitatively using flow cytometry, as shown in Fig. 7. After incubation with free PTX and PTX loaded nanomicelles for $6 \mathrm{~h}$, as presented in Fig. 7(A-G), the parent of internalized nanomicelles against Colon26 cells was $95.1 \%, 90.5 \%, 91.0 \%, 89.6 \%$ corresponding to $S 1, S 2, S 3$ and S4, respectively, which is almost in agreement with the changing trend of fluorescence intensity in Fig. 6. These results imply that the internalization of PTX loadedloaded S1-S4 nanomicelles occur through EPR effect, following dispersion, accumulation and PTX specific release. The apoptotic ratio of cells treated with free PTX and PTX loadedloaded micelles was also monitored through flow cytometry and shown in Fig. $7(\mathrm{H}) .23 .17 \%, 75.93 \%, 69.41 \%, 58.50 \%$ and $45.92 \%$ of apoptotic ratio are corresponding to PTX and PTX loadedloaded S1-S4, respectively, suggesting that the poor water solubility of PTX restrict indeed its ability to kill Colon26 cells. In contrast, the prepared S1-S4 nanomicelles could solubilize and realize controlled, targeted PTX release. Moreover, the increased cytotoxicity and PTX release rate of PTX loaded S1-S4 nanomicelles is proportional to the content of diselenide linkages.

\subsection{In vivo anticancer evaluation and histological analysis}

The biotoxicity and antitumor efficacy of PTX loadedloaded S1-S4 nanomicelles were assessed using Balb/c mice bearing 4T1 tumor. PTX loadedloaded S1 nanomicelles showed significantly tumor suppression and weight growth at a dosage of $5 \mathrm{mg} \mathrm{kg}^{-1}$ of PTX (Fig.8(A) and 8(B)), especially for S1, compared with the limited inhibition from PTX. Tumor inhibition ratio of free PTX, PTX loadedloaded S1, 
$\mathrm{S} 2$ is $18.24,45.27$ and $36.49 \%$, respectively. It is obviously observed that the different therapeutic effect of PTX

Figure 8 In vivo inhibition tumor efficacy of nanomicelles. Tumor volume (A) and body weight (B) of mice bearing 4T1 tumor treated with free PTX, normal saline, PTX-loaded S1 and S4 nanomicelles at a dose of $5 \mathrm{mg} \mathrm{kg}^{-1}$ over 15 days. Excised 4T1 solid tumors from different treatment groups on day 15 (C). H\&E stained major organ sections after $15 \mathrm{~d}$ treatment from different groups. (D)

toaded nanomicelles with diselenide and disulfide bonds against 4T1 tumor, indicating the burst and controllable PTX being released from nanomicellar assembly upon triggered by GSH, ROS and thermal stimulus. The excised tumor size from Fig. $8(\mathrm{C})$ is basically consistent with tumor volume using PTX and nanomicelles treatment.

The histological study in the H\&E staining method revealed that there is no obvious lesion can be observed in the group treated with PTX loaded nanomicelles, while the serious cardiotoxicity exhibited from PTX treated mice in Fig. 8(D), [30] reflecting that PTX entrapped nanomicelles are biocompatible in vivo and can release its cargo at the tumor site.

\section{Conclusions}

Thermal and redox responsive copolymers containing multiple diselenide/disulfide linkages and NIPAM units (PEG-alt-DXDP- $b$-PNIPAM, $X=S e$ or $S$ ) are prepared through alternative esterification and ATRP polymerization, in which the number of diselenide/disulfide linkage and PEG is eight via GPC detection. Considering the different response rate and mechanism of diselenide and disulfide bond, we developed the nanomicellar assemblies composed by PEG-alt-DSeDP- $b$-PNIPAM and PEG-alt-DSDP- $b$-PNIPAM with the mass ratios of 8:0, 5:3, 3:5 and 0:8, which were denoted as S1, S2, S3, S4, respectively. Further, PTX was introduced to obtain PTX loadedloaded S1-S4 nanomicelles. The marked thermal and response sensitivity were confirmed by DLS, TEM and UV-vis spectra. It was noteworthy that $72.90 \%$ of PTX was released from S1 nanomicelles within $23 \mathrm{~h}$ under the trigger of $10 \mathrm{mM} \mathrm{GSH}$ and high temperature, while $64.53 \%$ of PTX was released from S4 nanomicelles after $82 \mathrm{~h}$, indicating the burst and slow release for S1 and S4, respectively. As expected, the quantitative and qualitative cellular uptake and apoptosis illustrated that $\mathrm{S} 1$ killed cancerous cells during a short period of time, whereas S4 was more suitable for long-term treatment, S2 and S3 were intermediate. All results implied that the prepared nanomicellar assemblies achieved satisfactory effects according to the stage of tumor under the premise of avoiding the side effects of free PTX. Collectively, the design of nanoformula of copolymer combination with multiresponsiveness is of vital significance for cancer treatment with varied stages. PTX release behavior can be adjusted by developing nanomicellar assemblies composed of copolymers with the same composition and different response linkages, playing a pivotal role in the therapy of tumors at different stages.

\section{Declarations}




\section{Supporting Information}

Supporting Information is available online or from the author.

\section{Acknowledgements}

Not applicable.

\section{Authors' contribution}

Jingwen Xu and Xiangji Yan contributed equally to this work.

Jingwen Xu: Conceptualization, Methodology, Investigation, Data curation, Writing-Original draft, Visualization.

Xiangji Yan: Methodology, Investigation, Writing-Original draft, Visualization.

Yue Zhang: Investigation, Writing-Original draft, Visualization.

Mingzhen Zhang: Investigation, Data curation, Writing-Original draft.

Kangsheng Tu: Investigation.

Wen Shen: Investigation.

Zhaoqing Tian: Investigation.

Wei Zhao: Methodology, Investigation, Data curation, Writing-Original draft, Visualization.

\section{Funding}

This work was supported by the Specialized Scientific Research Fund Projects of Education Department of Shaanxi Province (21JK0530), Natural Science Fundamental Research Program of Shaanxi Province (2021JQ-550), Shaanxi Province Undergraduate Innovation \& Entrepreneurship Training Program (S202110708031), Natural Science Research Fund of Shaanxi University of Science and Technology (2019BJ-43), Key Research and Development Program of Shaanxi Province (2020SF-423 and 2020FP029).

\section{Ethics approval and consent to participate}

The mice were raised obey the standards of Laboratories Animal Center of Xi'an Jiaotong University, and all animal experiments were carried out and permitted by the guidelines from the Animal Ethics Committee of Xi'an Jiaotong University.

\section{Consent for publication}


All authors agree to be published.

\section{Availability of data and materials}

All data of this study are included in this work.

\section{Competing interests}

The authors declare no competing interest.

\section{References}

1. Cao Z, Li D, Wang J, Yang X. Reactive oxygen species-sensitive polymeric nanocarriers for synergistic cancer therapy. Acta Biomater. 2021, 130: 17-31.

2. Sabir F, Zeeshan M, Laraib U, Barani M, Rahdar A, Cucchiarini M, Pandey S. DNA Based and StimuliResponsive Smart Nanocarrier for Diagnosis and Treatment of Cancer: Applications and Challenges. Cancers. 2021, 13: 3396.

3. Ji W, Zhang T, Lu Z, Shen J, Zhang X. Synthesis and characterization of novel biocompatible nanocapsules encapsulated lily fragrance. Chinese Chem. Lett. 2019, 30: 739-42.

4. Hou Y, Bu W, Ai H, Lu Z-R, Lammers T. Stimuli-Responsive Nanotheranostics. Adv Healthc Mater. 2021, 10: 2100243.

5. Vaghasiya K, Ray E, Singh R, Jadhav K, Sharma A, Kha R, Katare OP, Verma RK. Efficient, enzyme responsive and tumor receptor targeting gelatin nanoparticles decorated with concanavalin-A for site-specific and controlled drug delivery for cancer therapy. Mat Sci Eng C. 2021, 123: 112027.

6. Zhang W, Lin W, Pei Q, Hu X, Xie Z, Jing X. Redox-Hypersensitive Organic Nanoparticles for Selective Treatment of Cancer Cells. Chem Mater. 2016 ,28: 4440-6.

7. Zheng P, Liu Y, Ding J. Targeted pH-responsive polyion complex micelle for controlled intracellular drug delivery. Chinese Chem Lett. $2020,31: 1178-82$.

8. Lu Z, Wang X, Zhang T, Zhang L, Yang J, Li Y, Shen J, Wang J, Niu Y, Xiao Z, et al. Cationic and temperature-sensitive liposomes loaded with eugenol for the application to silk. Chinese Chem Lett. 2020, 31: 3139-42.

9. Abandansari HS, Abuali M, Nabid MR, Niknejad H. Enhance chemotherapy efficacy and minimize anticancer drug side effects by using reversibly $\mathrm{pH}$ - and redox-responsive cross-linked unimolecular micelles. Polymer. 2017, 116: 16-26.

10. Pei Q, Hu X, Zhou J, Liu S, Xie Z. Glutathione-responsive paclitaxel dimer nanovesicles with high drug content. Biomater Sci. 2017, 5: 1517-21.

11. Ding C, Wu H, Yinc Z-Z, Gao J, Wu D, Qin Y, Kong Y. Disulfide-cleavage- and pH-triggered drug delivery based on a vesicle structured amphiphilic self-assembly. Mat Sci Eng C. 2020, 107: 10366.

12. He Y, Guo S, Zhang Y, Liu Y, Ju H. Near-Infrared Photo-controlled Permeability of a Biomimetic Polymersome with Sustained Drug Release and Efficient Tumor TherapyTherapy. ACS Appl Mater 
Inter. 2021, 13: 14951-63.

13. Hamoudi-Ben Yelles MC, Tran Tan V, Danede F, Willart JF, Siepmann J. PLGA implants: How Poloxamer/PEO addition slows down or accelerates polymer degradation and drug release. J Control Release. 2017, 253: 19-29.

14. Zhang Y, Uthaman S, Song W, Eom KH, Jeon SH, Huh KM, Babu A, Park I-K, Kim I. MultistimuliResponsive Polymeric Vesicles for Accelerated Drug Release in Chemo-photothermal Therapy. ACS Biomater Sci Eng. 2020, 6: 5012-23.

15. Cheng R, Feng F, Meng F, Deng C, Feijen J, Zhong Z. Glutathione-responsive nano-vehicles as a promising platform for targeted intracellular drug and gene delivery. J Control Release. 2011, 152: 212.

16. He M, Yu L, Yang Y, Zou B, Ma W, Yu M, Lu J, Xiong G, Yu Z, Li A. Delivery of triptolide with reductionsensitive polymer nanoparticles for liver cancer therapy on patient-derived xenografts models. Chinese Chem Lett. 2020, 31: 3178-82.

17. Hailemeskel BZ, Addisu KD, Prasannan A, Mekuria SL, Kao C-Y, Tsai H-C. Synthesis and characterization of diselenide linked poly(ethylene glycol) nanogel as multi-responsive drug carrier. Appl Surf Sci. 2018, 449: 15-22.

18. Yan Q, Yang Y, Chen W, Hu J, Yang D. Construction of polymer-paclitaxel conjugate linked via a disulfide bond. Mat Sci Eng C. 2016, 58: 580-5.

19. Deepagan VG, Kwon S, You DG, Nguyen VQ, Um W, Ko H, Lee H, Jo D-G, Kang YM, Park JH. In situ diselenide-crosslinked polymeric micelles for ROS-mediated anticancer drug delivery. Biomaterials. 2016, 103: 56-66.

20. Zheng L, Li C, Huang X, Lin X, Lin W, Yang F, Chen T. Thermosensitive hydrogels for sustained-release of sorafenib and selenium nanoparticles for localized synergistic chemoradiotherapy. Biomaterials. 2019, 216: 119220.

21. Yang M, Lee SY, Kim S, Koo JS, Seo J-H, Jeong DI, Hwang CR, Lee J, Cho H-J. Selenium and dopamine-crosslinked hyaluronic acid hydrogel for chemophotothermal cancer therapy. J Control Release. 2020, 324: 750-64.

22. Zhang W, Lin W, Pei Q, Hu X, Xie Z, Jing X. Redox-Hypersensitive Organic Nanoparticles for Selective Treatment of Cancer Cells. Chem Mater. 2016, 28: 4440-6.

23. Zuo S, Sun B, Yang Y, Zhou S, Zhang Y, Guo M, Sun M, Luo C, He Z, Sun J. Probing the Superiority of Diselenium Bond on Docetaxel Dimeric Prodrug Nanoassemblies: Small Roles Taking Big Responsibilities. Small. 2020, 162005039.

24. Xu J, Ge X, Lv L, Xu F, Luo Y. Dual-Stimuli-Responsive Paclitaxel Delivery Nanosystems from Chemically. Conjugate Self-assemblies for Carcinoma Treatment. Macromol Rapid Comm. 2018, 39: 1800628.

25. Xu J, Yan X, Ge X, Zhang M, Dang X, Yang Y, Xu F, Luo Y, Li G. Novel multi-stimuli responsive Functionalized PEG-based co-delivery nanovehicles toward sustainable treatments of multidrug resistant tumor. J Mater Chem B. 2021, 9: 1297-314. 
26. Mai NXD, Nguyen T-HT, Vong LB, Dang M-HD, Nguyen TTT, Nguyen LHT, Ta HKT, Nguyen T-H, Phan TB, Doan TLHJMS. Tailoring chemical compositions of biodegradable mesoporous organosilica nanoparticles for controlled slow release of chemotherapeutic drug. Mater Sci Eng C. 2021, 127: 112232.

27. Hanaor D, Michelazzi M, Leonelli C, Sorrell CC. The effects of carboxylic acids on the aqueous dispersion and electrophoretic deposition of $\mathrm{ZrO}_{2}$. Eur J Ceram Soc. 2012, 32: 235-324.

28. Sun T, Jin Y, Qi R, Peng S, Fan B. Oxidation responsive mono-cleavable amphiphilic diblock micelles labeled with a single diselenide. Polym Chem. 2013, 4: 4017-23.

29. Chang J, Li Y, Wang G, He B, Gu Z. Fabrication of novel coumarin derivative functionalized polypseudorotaxane micelles for drug delivery. Nanoscale. 2013, 5: 813-20.

30. Yin T, Wang L, Yin L, Zhou J, Cao M. Co-delivery of hydrophobic paclitaxel and hydrophilic AURKA specific siRNA by redox-sensitive micelles for effective treatment of breast cancer. Biomaterials. 2015, 61: 10-25.

\section{Figures}


A

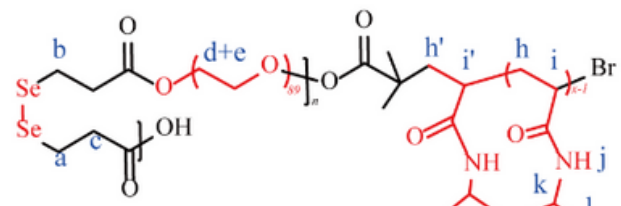

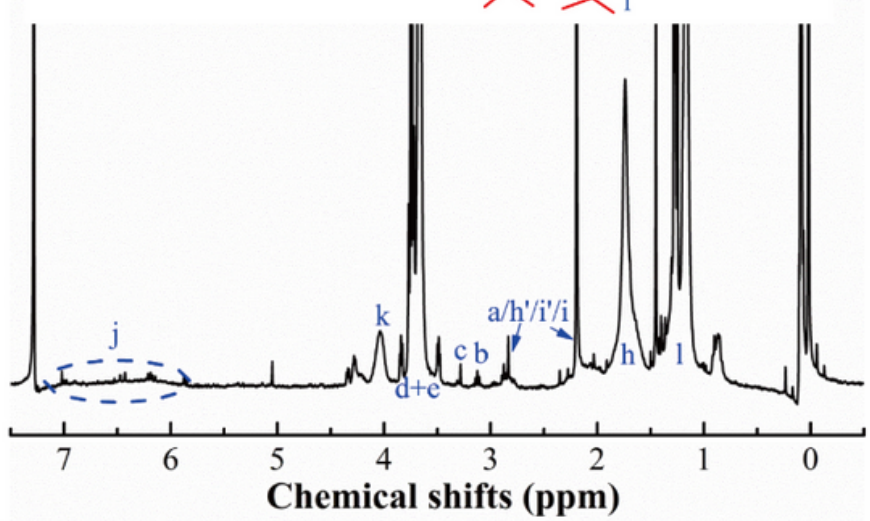

C

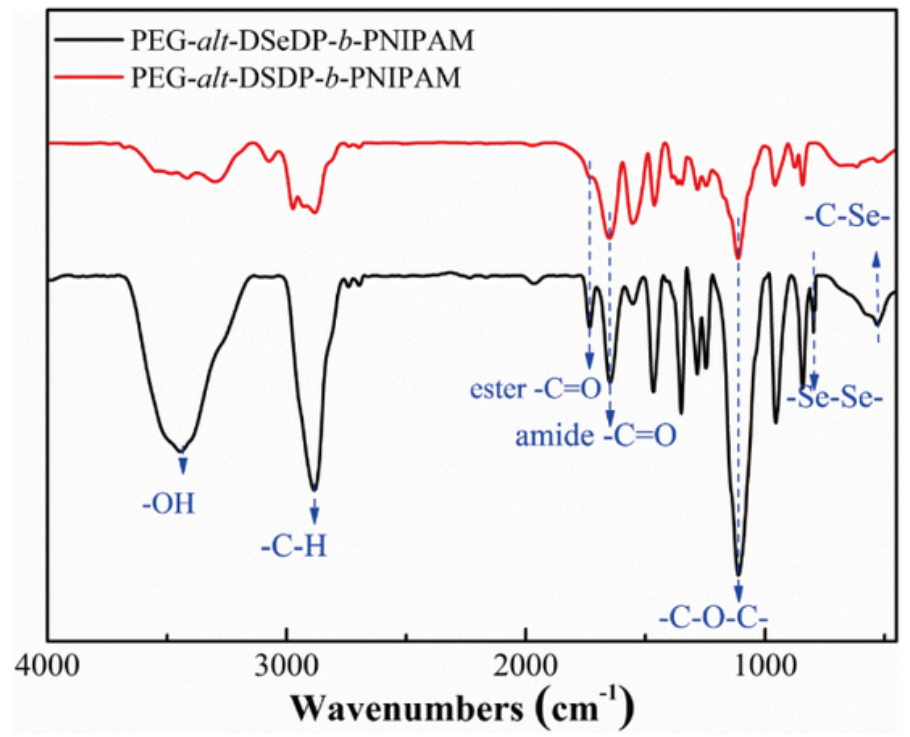

B

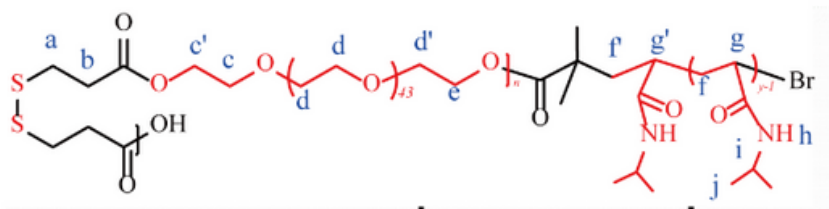

D

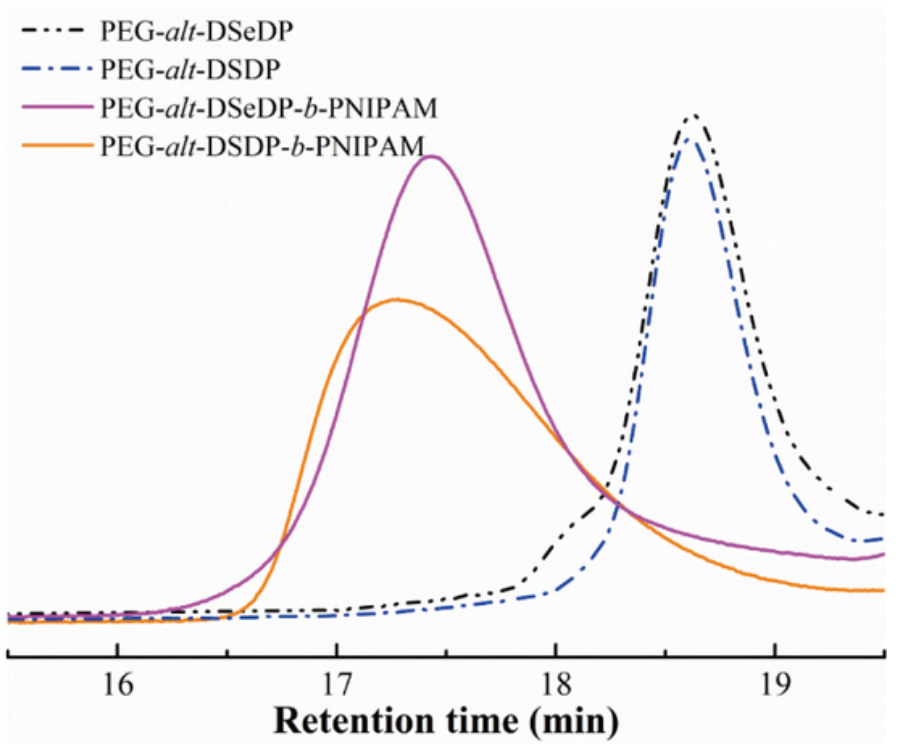

Figure 1

1H NMR (A, B), FTIR (C) spectra and GPC curves (D) of PEG-alt-DSeDP-b-PNIPAM and PEG-alt-DSDP-bPNIPAM. 
A

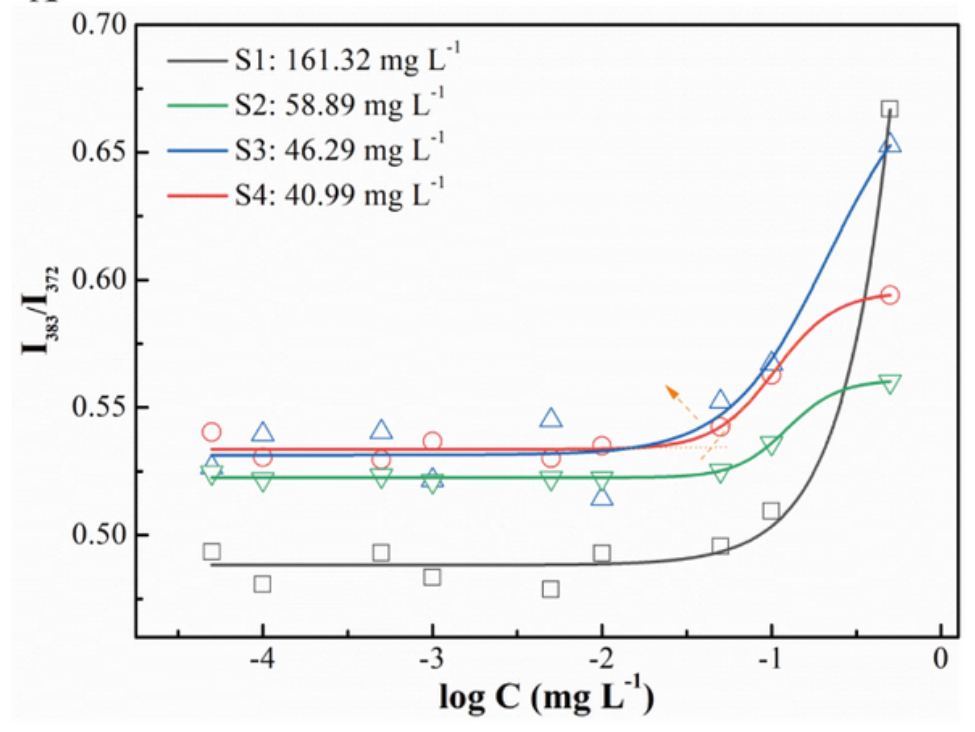

B

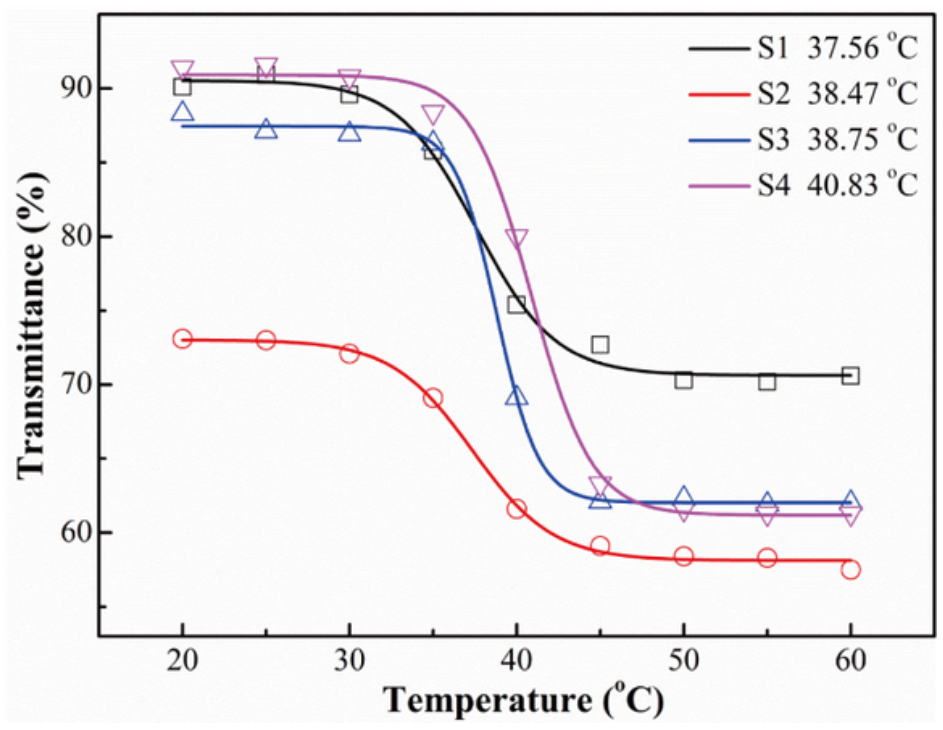

Figure 2

(A) Plots of fluorescence intensity ratio I383/I372 from pyrene emission spectrum vs. logarithm of the blank nanomicellar concentration. (B) Temperature-dependent transmittance changes of nanomicelles. (S1, S2, S3 and S4 were composed of PEG-alt-DSeDP-b-PNIPAM and PEG-alt-DSDP-b-PNIPAM according to the mass ratios of $8: 0,5: 3,3: 5$ and $0: 8$, respectively.)
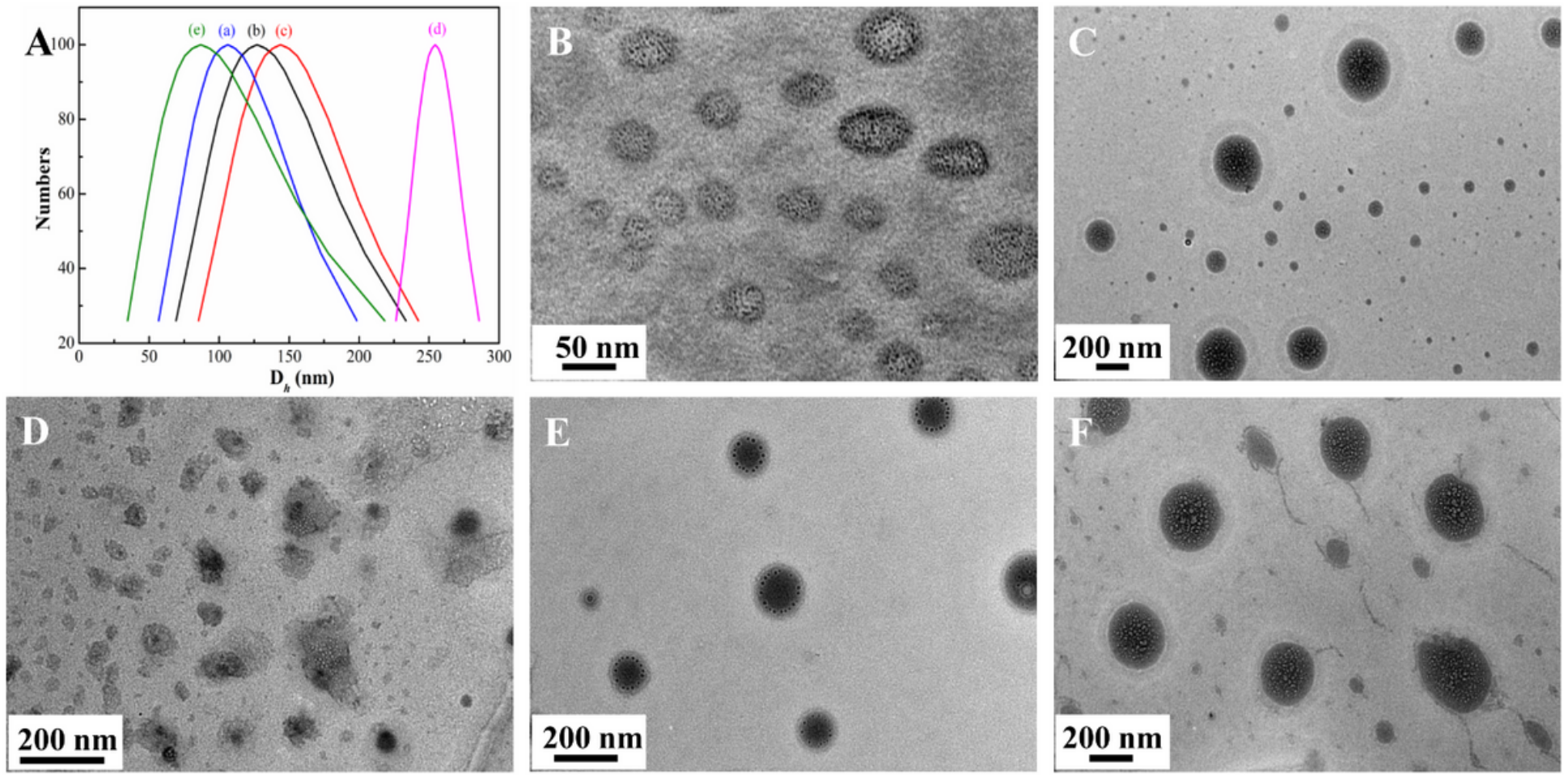

\section{Figure 3}

Size dispersion of S1 nanomicelles treated with 0 mM GSH (a), 10 mM GSH ((b), (c), (d)) and $100 \mu \mathrm{M}$ $\mathrm{H} 202$ (e) within $0.5 \mathrm{~h}(\mathrm{~b}), 4 \mathrm{~h}(\mathrm{c}), 24 \mathrm{~h}(\mathrm{~d}, \mathrm{e})$ at $25 \mathrm{oC}$ from DLS (A). TEM photographs of S1 blank (B), 
reduced by $10 \mathrm{mM} \mathrm{GSH}$ (C), oxidized by $100 \mu \mathrm{M} \mathrm{H} 2 \mathrm{O} 2$ (D), PTX loaded (E) and thermo, GSH induced released (F) S1 nanomicelles.

A

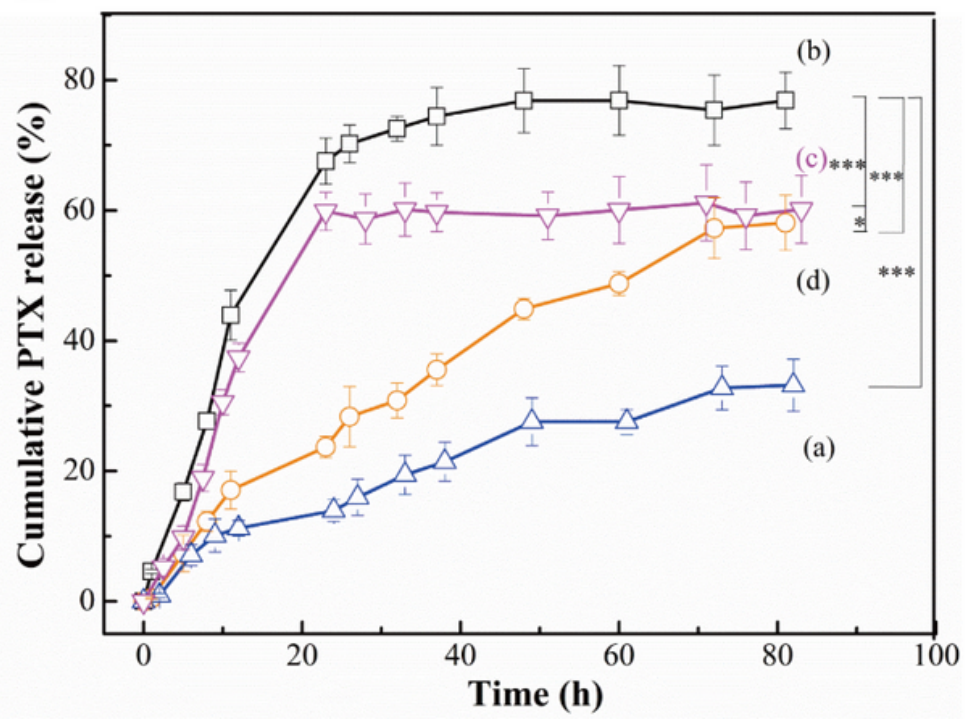

B

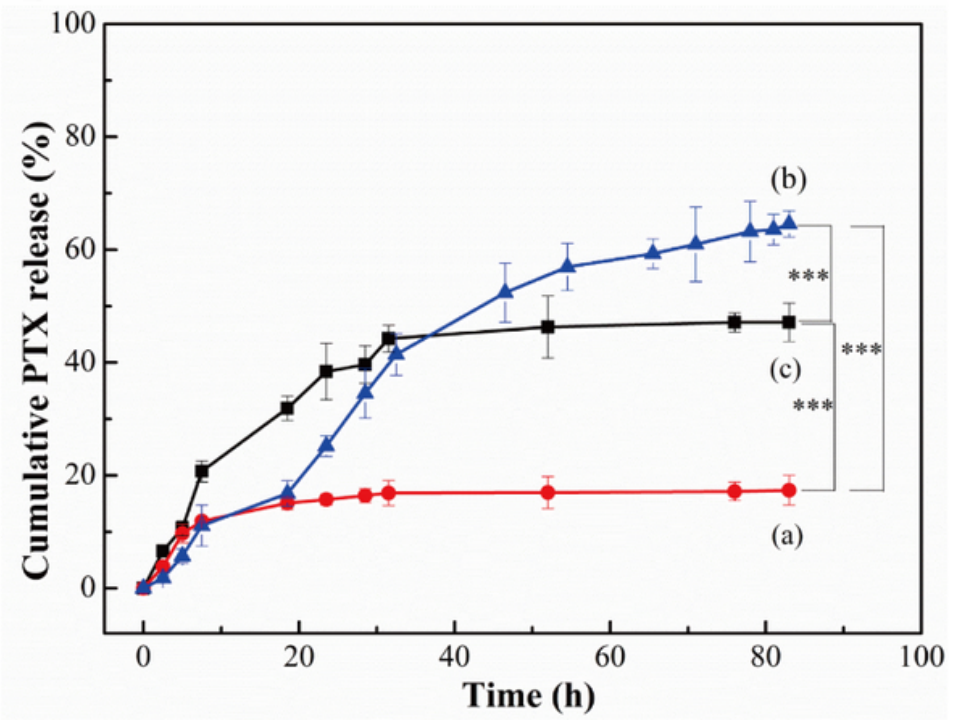

Figure 4

In vitro PTX release profiles of S1 (A) and S4 (B) nanomicelles in pH 7.4 PBS at 37 oC (a), in pH 5.6 PBS containing $10 \mathrm{mM} \mathrm{GSH}$ at $42 \mathrm{oC}$ (>LCST) (b), in pH 5.6 PBS with $10 \mathrm{mM} \mathrm{GSH}$ at $35 \mathrm{oC}$ (<LCST) (c) and in pH 5.6 PBS with $100 \mu \mathrm{M} \mathrm{H} 2 \mathrm{O} 2$ at 40 oC (d) for $82 \mathrm{~h}$.

A

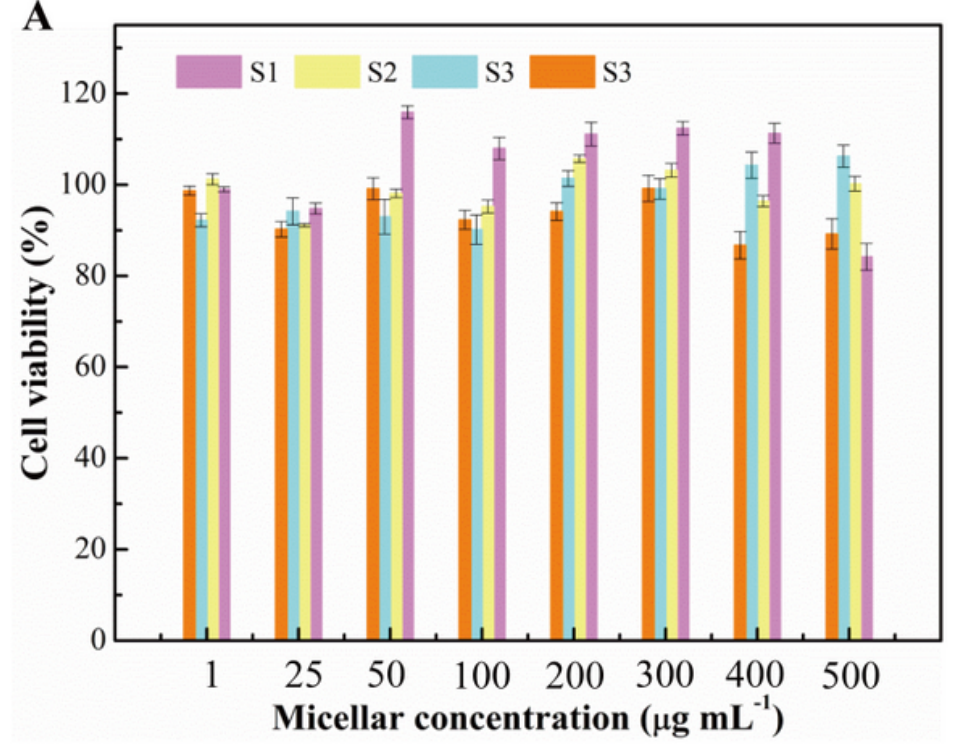

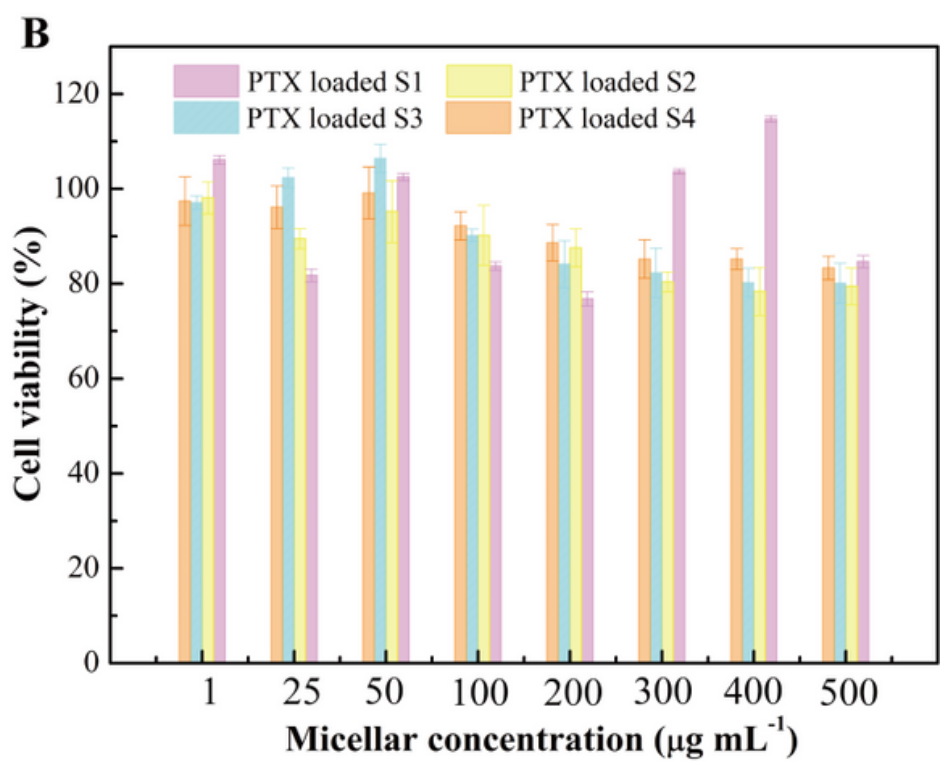

\section{Figure 5}

The viability of L929 cells treated with blank (A) and PTX loaded (B) S1-S4 nanomicelles. 


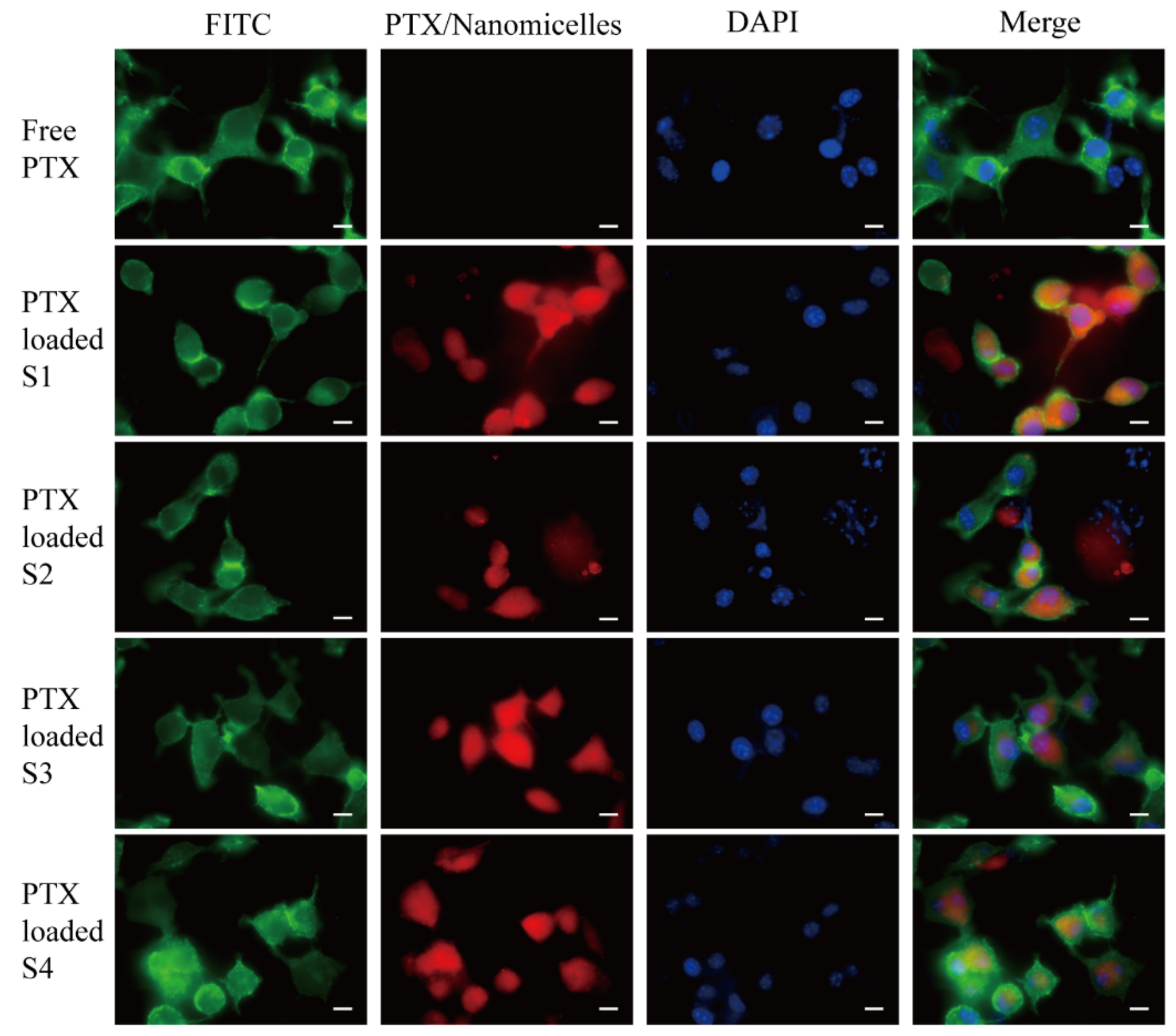

Figure 6

Qualitative analysis of cellular uptake of PTX by CLSM in Colon26 cells incubated with PTX, PTX loaded S1-S4 nanomicelles after $6 \mathrm{~h}$. (100x) 
A

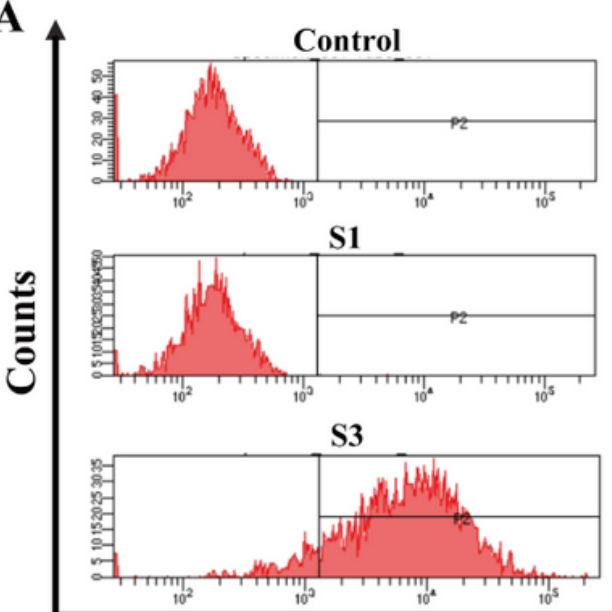

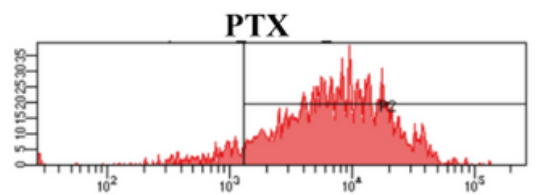
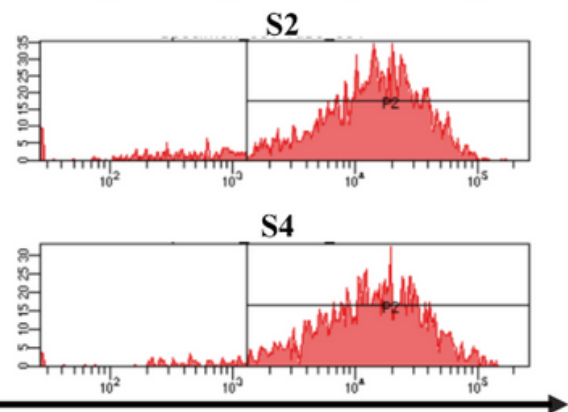

PI
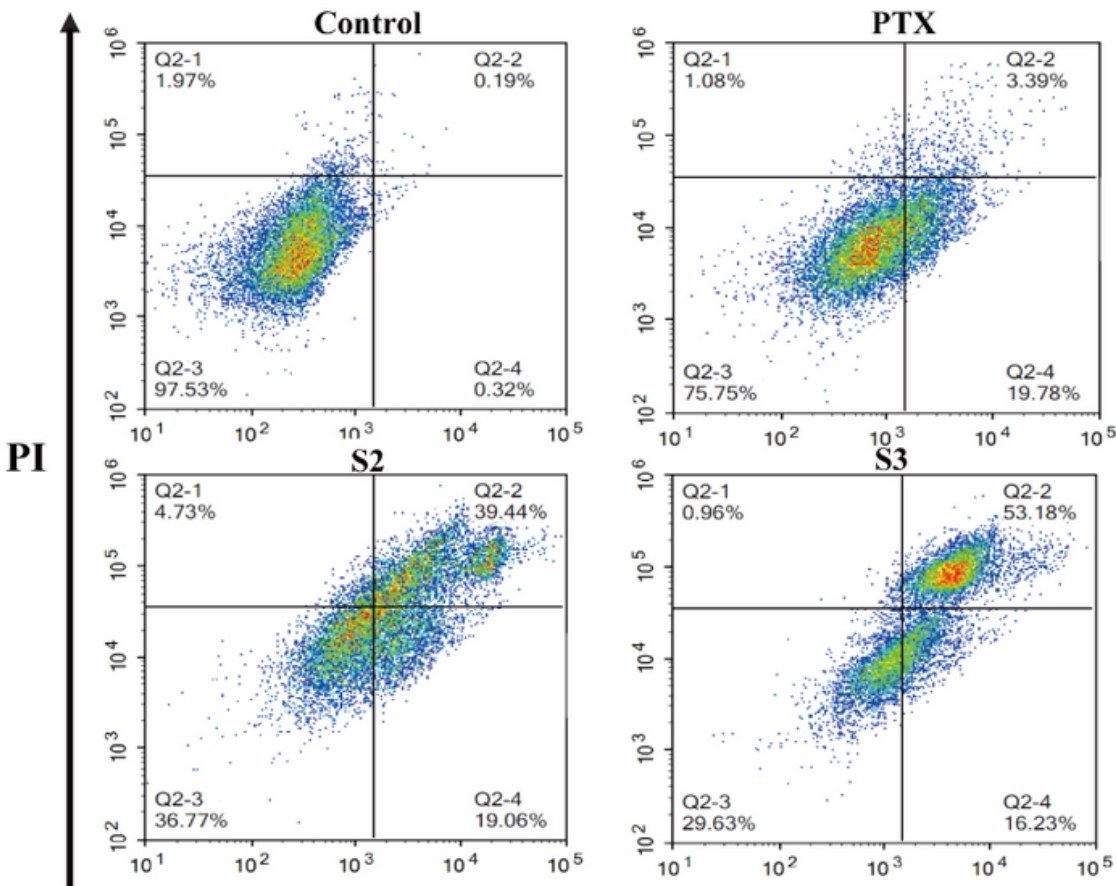

B

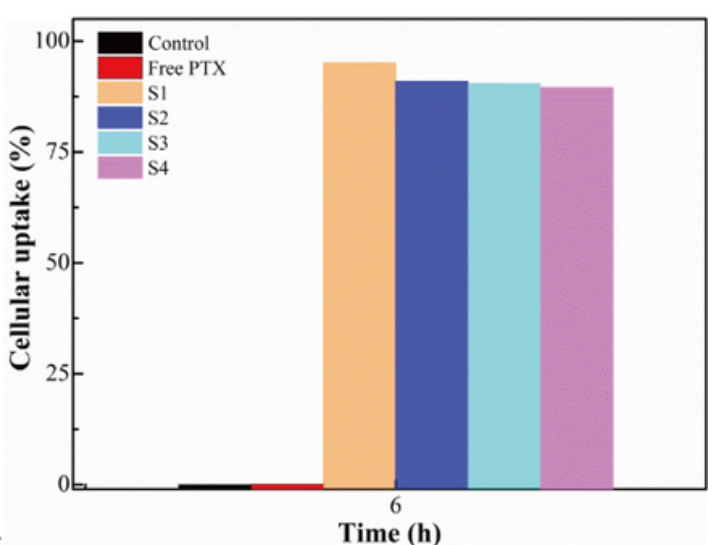

C
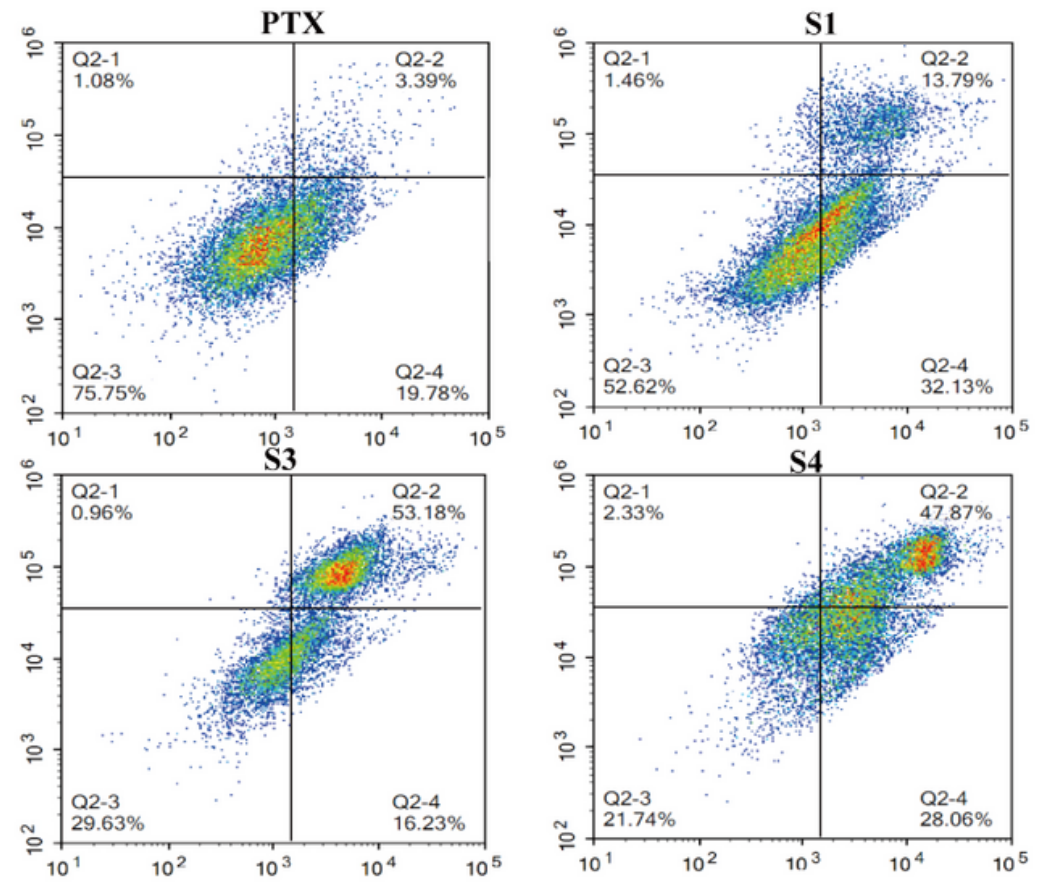

Annexin V-FITC

\section{Figure 7}

Quantitative analysis of cellular uptake of PTX loaded nanomicelles by FCM in Colon26 cells (A-G). Cell apoptosis of free PTX and PTX loadedloaded S1-S4 (H) nanomicelles in Colon26 cells.

\section{Figure 8}

In vivo inhibition tumor efficacy of nanomicelles. Tumor volume (A) and body weight (B) of mice bearing 4T1 tumor treated with free PTX, normal saline, PTX-loaded S1 and S4 nanomicelles at a dose of $5 \mathrm{mg}$ kg-1 over 15 days. Excised 4T1 solid tumors from different treatment groups on day 15 (C). H\&E stained major organ sections after $15 \mathrm{~d}$ treatment from different groups. (D) 


\section{Supplementary Files}

This is a list of supplementary files associated with this preprint. Click to download.

- Graphicabstract.docx

- schema1.png

- SupportingInformation.docx 